\title{
Análisis de la Gestión en la Producción y Comercialización de Cacao en la Provincia de Santo Domingo de los Tsáchilas
}





\section{Resumen}

El presente trabajo expone los principales resultados de una investigación realizada en la provincia de Santo Domingo de los Tsáchilas sobre las condiciones y causas que determinaron la obtención de resultados ineficientes e ineficaces en la producción y comercialización del cacao en el último lustro, como premisa para implementar cambios estratégicos en la concepción de la matriz productiva en ese rubro de producción en la provincia.

Como objetivo de la investigación se definió evaluar la gestión en la producción y comercialización de los productores de cacao de Santo Domingo, para lo cual se investigó en 30 asociaciones de cacaoteros con 729 asociados, aplicándose herramientas como cuestionarios, entrevistas y focus group tanto en esas organizaciones como en las instituciones gubernamentales e intermediarios.

La escasa gestión de los cacaoteros por la incidencia de la desarticulación de los actores ha hecho que sus niveles de vida sean bajos, por lo que se propone una concertación de una mesa de cacao enmarcada en los principios de asociatividad.

Palabras clave: asociatividad, comercialización, cacao, gestión, producción

\section{Abstract}

This work presents the main results of a research made in the province of Santo Domingo de los Tsáchilas about the conditions and causes which determined getting inefficient and ineffective results in both production and marketing of cocoa in the last five years, which helped to implement strategies changes in the conception of the productive matrix production in the province.

To evaluate the management in the production and marketing of cocoa farmers of Santo Domingo was defined as research objective, so 30 associations and 729 members of cocoa were investigated by applying questionnaires, interviews and focus groups in these organizations and government institutions and intermediaries too.

The poor management made by cocoa producers which is based in the impact of dismantling of the actors has made living standards getting low, so a concerted cocoa table framed based on the principles of partnership is proposed.

Keywords: Associativity, cocoa, management, marketing, production. 


\section{INTRODUCCIÓN}

En el año 2012 Santo Domingo de los Tsáchilas registró una superficie total sembrada de cacao de 19.837 hectáreas, de las cuales se cosecharon 13.603 hectáreas según datos del Ministerio de Agricultura, Ganadería, Acuacultura y Pesca (MAGAP). La provincia ocupa el quinto lugar como región productora de cacao a nivel nacional, lo que ha hecho que Santo Domingo haya sido reconocida otorgándole la sede de actos oficiales destacados del sector (El Comercio, 2013).

La actividad de producción y comercialización del cacao en los mercados nacionales e internacionales por parte de las pequeñas y medianas asociaciones cacaoteras de Santo Domingo podría constituir un área productiva capaz de transformar la situación económica de la población de esta provincia; sin embargo según el Censo Económico y de Vivienda 2013 del Instituto Nacional de Estadística y Censos (INEC) el $30,14 \%$ de sus habitantes son pobres por ingresos, y el $74,36 \%$ lo son por necesidades básicas insatisfechas. Esta situación es aún más grave en las cinco parroquias rurales (Luz de América, San Jacinto, La Concordia, Puerto Limón y Santa María del Toachi), con indicadores que sitúan a sus pobladores como pobres $(89,65 \%)$ o en la extrema pobreza $(33,83 \%)$ (INEC, 2013).

Desde la óptica de la estructura económica existe una dispersión de los productores, tanto en el patrimonio (las tierras) como en el cultivo mismo siendo éste un factor que condiciona el carácter individual de la producción y que al mismo tiempo dificulta la formación de una fuerza de masa propia de la asociatividad en cualquiera de sus manifestaciones. Todo ello condena a un alto nivel de vulnerabilidad económica a esos actores de la producción de cacao de la provincia.

Por otra parte, la legalización de las asociaciones necesita conocimientos de los procesos que requiere el Gobierno. Precisamente la carencia de líderes en estas asociaciones, es que hace que se encuentren legitimadas tan sólo el $34 \%$ de ellas, de acuerdo con los datos del GAD de Santo Domingo (2012).

Si bien el proceso de producción es poco favorable en sí mismo, el transporte, distribución y circulación la situación es más grave. La existencia de centros de acopio privados monopólicos o eventualmente oligopólicos favorece la imposición de un nivel de precios que no sólo no garantiza un incremento en la producción del producto, sino que la desestimula: únicamente el $5 \%$ de los ingresos que genera el cacao llega al productor (GAD de Santo Domingo, 2012).

Además, se observa un bajo nivel de diversificación de la producción en el territorio, al constatar que más del $90 \%$ del cacao comercializado se oferta como materia prima, existiendo únicamente una pequeña empresa familiar que realiza el proceso de elaboración de pasta de chocolate. Ello hace económicamente dependientes a los agricultores de los sectores exógenos transformativos (GAD de Santo Domingo, 2012).

Ante esta realidad el presente trabajo de investigación tiene por objetivo identificar las necesidades, situación y nivel de gestión (eficiencia y eficacia) de las asociaciones productoras de cacao de Santo Domingo, y cómo se relacionan con las entidades gubernamentales -Gobierno Provincial, Instituto Nacional de Investigaciones Agropecuarias (INIAP) y MAGAP-, intermediarios y ONG's, todo ello con el fin de desarrollar estrategias que articulen a las asociaciones del sector cacaotero de cara a la mejora del nivel de vida de sus asociados. Esta investigación se encuentra incluida dentro del marco del proyecto Mejoramiento de la cadena productiva del cacao en Santo Domingo, impulsado por el GAD Provincial y la Unión Europea,el cual inicio en 2012 en una primera etapa que está previsto finalice en 2015.

Este trabajo se llevó a cabo por parte de los investigadores durante el año 2013 y parte del año 2014, estando todos los datos recabados referidos a 2013.

Enlarevisiónbibliográficaseencontraronexperienciasque sirven de referentes para nuestras unidades productivas basadas en la filosofía asociativa (Mónica G. Liendo y Adriana M. Martínez, 2001; Colmenarez y Delgado, 2003; Narváez, Fernández G., Gutiérrez C., Revilla J. y Pérez, 2009; Luckesh, 2000; Cervilla, 2007; Rosales, 1997). Así, por ejemplo, la Asociación de Pequeños Productores de Talamanca (APPT) en Costa Rica, que agrupa a más de 50 asociaciones que comercializa alrededor de 300 toneladas de cacao al año; o CONACADO, una 
entidad de República Dominicana que cuenta con 9.000 pequeños productores que llegan a representar el $25 \%$ de la producción nacional.

Liendo y Martínez (2001) citan a Poliak (2001), quien explica que la asociatividad, es una estrategia de colaboración colectiva que se vincula a negocios concretos, de forma que se constituye en herramienta al servicio de un negocio. Además detalla que las empresas en ese contexto "desarrollan un esfuerzo colectivo para la concreción de objetivos comunes, que pueden ser muy disímiles, desde comprar en común programas de investigación y desarrollo o mejores posicionamientos en la cadena de valor para su esquema de negociación" (Liendo \& Martínez, 2001, pág. 311).

Todos estos modelos proponen una estructura orgánica funcional para mejorar la gestión de estas unidades productivas, pero este tipo de estructura no resolverá, por si sola el problema objeto de estudio en el presente trabajo; en cambio la integración de los actores y las interrelaciones sistemáticas y sistémicas con el entorno sí garantizan una gestión integral para alcanzar el buen vivir.

Algunos aspectos de esta investigación quedan pendientes de profundizar, como son las relaciones entre los productores y los centros de acopio de exportación, que ofrecerían interesantes posibilidades de negociaciones, de forma que, por ejemplo, pudieran funcionar como un consorcio de exportación. Serían múltiples las formas de negociaciones que se podrían plantear para generar una mayor productividad $y$ calidad en las asociaciones de cacaoteros de la provincia de Santo Domingo.

\section{MÉTODOS Y MATERIALES}

En la gestión de la producción y comercialización de cacao en Santo Domingo existen varios actores que inciden en el resultado final del proceso, pero aquí nos centraremos en los socios de cada asociación de cacaoteros con el objetivo de medir el grado de aislamiento de los agricultores y evaluar cómo este factor incide en el mayor o menor grado de eficiencia y eficacia de la gestión de las asociaciones estudiadas.
Para ello fue necesario elaborar dos baterías de cuestionarios, una sobre aislamiento y otra sobre las relaciones intersocios- para los agricultores. La población -determinada gracias a un censo realizado a partir del trabajo conjunto con estudiantes de últimos niveles de la Facultad de Ciencias Económicas de la Universidad Tecnológica Equinoccial sede Santo Domingo y del GAD Provincial- está formada por 30 asociaciones con una suma total de 729 socios, de los cuales el $58,76 \%$ son hombres y el $41,24 \%$ mujeres. Toda la población fue sometida a las preguntas de las dos baterías de cuestionarios.

Para abordar las relaciones existentes entre los agricultores y las instituciones del gobierno provincial se llevaron a cabo entrevistas al gerente del proyecto GAD-UE, así como a dos técnicos del GAD provincial especializados en manejo del cacao y asociatividad. Igualmente se realizaron entrevistas a responsables de los siete centros de acopio de cacao para exportación situados en la provincia, al presidente de la Asociación de Cacao de Santo Domingo de los Tsáchilas, y con un investigador-agricultor altamente especializado en el manejo del cultivo que nos ocupa.

La situación de los agricultores de cacao en lo referente a la planificación se abordó desde tres ángulos: la falta de planeación de las variedades de cacao a sembrar y la superficie de cultivo dedicado a cada una; la ausencia de una guía de planeación en la propia siembra; y la carencia de una planificación desde el punto de vista del crédito y el uso que se va a dar al recurso económico.

\section{RESULTADOS}

\section{Planificación}

La planificación en cuanto a la siembra por parte de los socios de las asociaciones es escasa y no está coordinada adecuadamente con el plan puesto en marcha desde el Gobierno Provincial Descentralizado (GAD). En las baterías de cuestionarios, los agricultores manifiestan que si se les entregan plantas de la variedad adecuada para cada zona y con un descuento del $50 \%$ sobre el valor real de mercado desde el gobierno autónomo descentralizado; pero estas afirmaciones contrastan con la realidad observada en la investigación de campo 
realizada. Pese a existir asesoramiento y capacitación desde el GAD provincial (el $52 \%$ asegura acudir a las reuniones de formación), el $48 \%$ de los agricultores dice no sembrar de acuerdo a ninguna planificación u orientación de las instituciones encargadas de asesorar. Cabe mencionar que los temas impartidos en las capacitaciones son manejos de cultivos (68\%), poscosecha $(20 \%)$ y comercialización ( $12 \%)$, de lo que cabe deducir que la mayoría de las horas de formación se centra precisamente en labores de siembra, podas, etc., pudiendo concluir que los agricultores carecen de la formación adecuada en estos aspectos.

Si nos referimos a las hectáreas cultivadas, de acuerdo con los datos obtenidos en 2013 la variedad de cacao Nacional en forma de monocultivo ocupó 988,23 hectáreas, mientras que 362,81 hectáreas fueron sembradas de manera asociada a otros cultivos (maracuyá, plátano, yuca), y otras 736,5 hectáreas fueron proyectadas (terrenos preparados para ser sembrados de cacao a corto plazo). Además para el mismo año $1.289,3$ hectáreas fueron sembradas con la variedad CCN51 en monocultivo, 240,74 hectáreas se cultivaron asociadamente con otros productos y sólo se proyectaron 82,75 hectáreas. Desde el GAD Provincial se busca fomentar la siembra de la variedad Nacional a través de las capacitaciones que imparte a los agricultores de las asociaciones, pero la realidad indica que los productores siguen apostando por la CCN51 debido a que ésta tiene un menor tiempo de puesta en producción, mayor rendimiento y es menos exigente en cuidados que la variedad Nacional.

Por último, la búsqueda y posterior gestión de créditos por parte de las asociaciones podemos calificarla de nula de acuerdo a la línea base levantada. La carencia de líderes dentro de las asociaciones provoca cambiar por: la ausencia de gestión para solicitar créditos que puedan beneficiar a sus asociados. Ante esta situación los agricultores socios de las mismas en un gran porcentaje acuden de forma particular al Banco Nacional de Fomento(BNF) para conseguir financiación, pese a que las condiciones de acceso al crédito son menos favorables para ellos de lo que serían si fueran las propias asociaciones las que solicitasen la financiación, gracias a la legislación nacional en vigor sobre acceso a crédito para asociaciones y cooperativas. En su mayoría los fondos obtenidos (ver Gráfico 1) se dedican a aspectos como la resiembra, dejando la inversión en poscosecha en un marginal $1 \%$ del total, cuando la poscosecha es una fase crítica en la que un tratamiento erróneo o no adecuado de las pepas puede disminuir la calidad y por ende el valor final del producto. El escaso acceso al crédito como cooperativas, la carestía de las condiciones de la financiación al acudir a las entidades bancarias a título individual y el desequilibrio en la distribución de esos recursos económicos entre las distintas fases del proceso de cultivo afectan sin duda a las posibilidades de mejora de las plantaciones en productividad, calidad y rentabilidad.

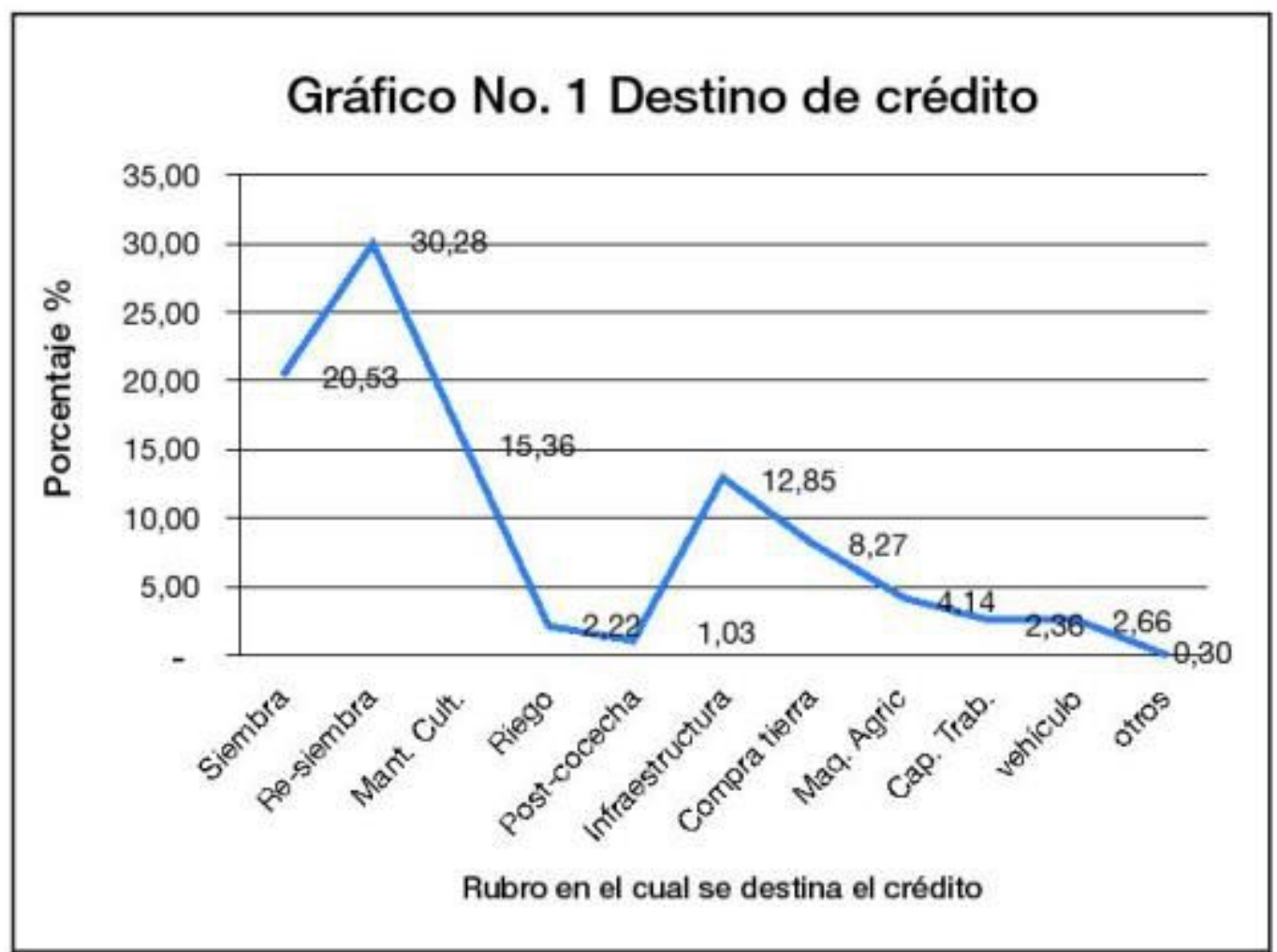

Fuente: Línea Base GAD

\section{Organización}

Al hablar del aspecto organizacional encontramos que las asociaciones del sector en la provincia de Santo Domingo carecen de organización estructural pese a que el estado lo establece en el modelo de Economía Popular y Solidaria. La mayoría de los socios de estas asociaciones negocian por su propia cuenta con intermediarios y comerciantes locales la venta de su producción. Se estima que el $90 \%$ de la comercialización del cacao generado en la provincia se lo hace de la manera descrita. 


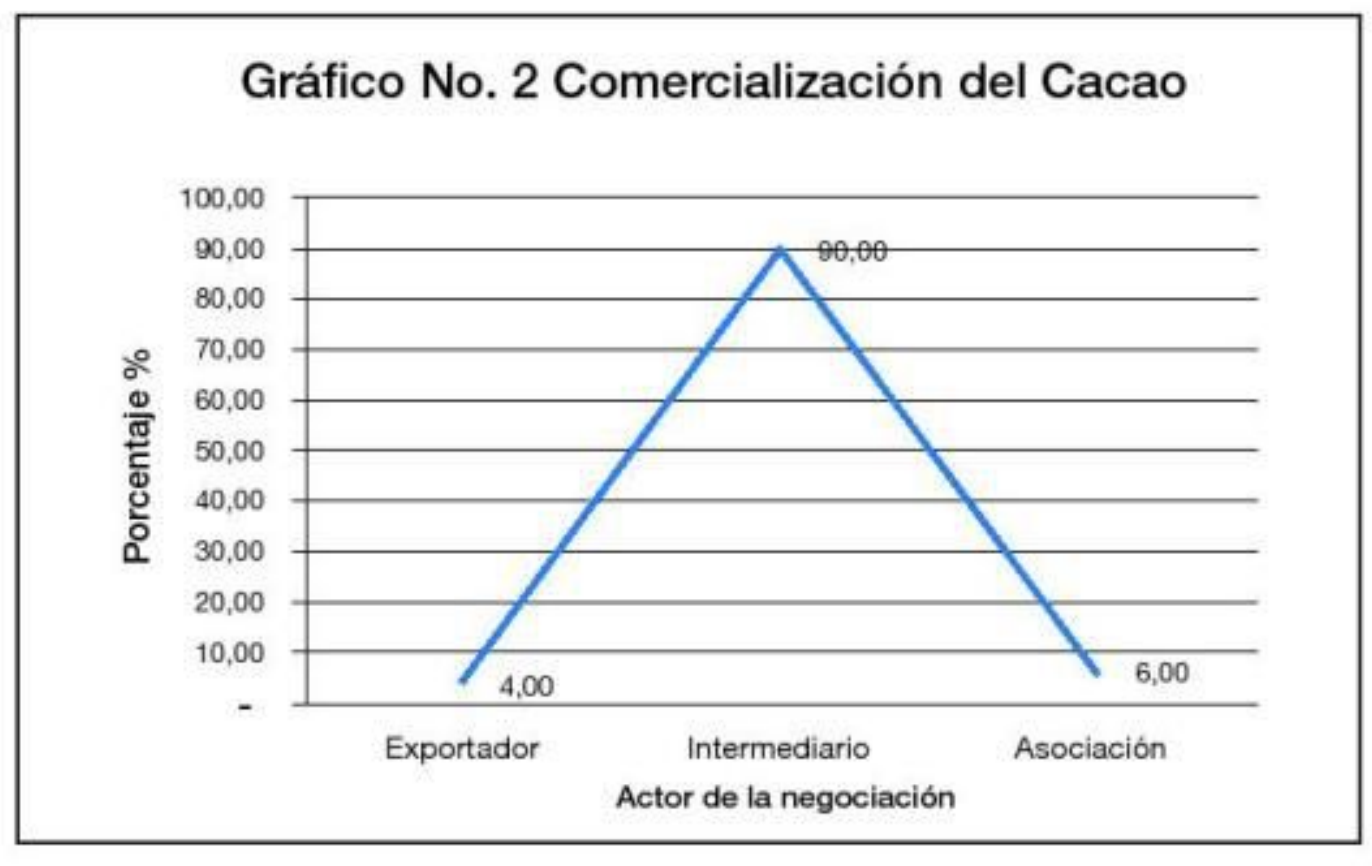

Fuente: Línea Base GAD

La asociatividad ha sido la estrategia más poderosa puesta en marcha por otras iniciativas de unidades productivas de cacao de la región centro y sur americana para competir y sobrevivir en una competencia desigual. Diversas experiencias recopiladas en nuestra revisión bibliográfica centradas fundamentalmente en los países andinos y de Centroamérica muestran cómo dentro de la concepción de cadenas agro-productivas se dan diálogos y acuerdos de concertación que mejoran el poder de negociación de los agricultores frente al resto de actores de la cadena de valor del cacao.

En el caso que nos ocupa se ha observado en cambio una falta de organización dentro de la estructura de las distintas asociaciones a la hora de poner en el mercado la producción obtenida. Pese a que en cada una de las asociaciones se ha establecido un organigrama donde se plasma cuál es la estructura clara, con puestos y responsabilidades definidas, dicho esquema no tiene un reflejo en la práctica. Así, la mayoría de presidentes de cada asociación no posee una actitud proactiva a la hora de, por ejemplo, buscar negociaciones con potenciales compradores o intermediarios para intentar llegar a acuerdos de compra-venta más beneficiosos para sus asociados. El no cumplimiento de las funciones de los distintos cargos directivos produce que la organización de las asociaciones no funcione y, con ello, que los socios no vean beneficios que les reporta, al ser parte de la entidad, lo que los conduce a negociar por ellos mismos la venta de su cosecha en una posición de claro desequilibrio respecto a los compradores.

Parte del problema también lo compone el insuficiente proceso de concertación de los objetivos entre los propios socios de las asociaciones, así como la poca efectividad de las distintas instituciones y organizaciones del gobierno provincial en sus intentos de integrar la gestión de los pequeños y medianos productores por los objetivos estratégicos del desarrollo local. En esta falencia tiene mucho que ver el nivel educativocultural de los propios agricultores, como analizaremos a continuación.

\section{Liderazgo}

En las asociaciones analizadas se ha encontrado una clara falta de liderazgo, evidenciado en el hecho de que de las 30 entidades que existen en la provincia sólo 14 están legalizadas de acuerdo a lo contenido en la Ley Orgánica de la Economía Popular y Solidaria (EPS), estando, a marzo del 2014, el resto en proceso de legalización. Pese a que el GAD provincial pone a disposición de las asociaciones un técnico con experiencia en conformación y gestión de entidades asociativas, muchos de los presidentes de las agrupaciones de agricultores ni siquiera asisten a las reuniones convocadas porel profesional, algo que podría deberse a un desinterés. En este sentido también se debe tomar en cuenta la cultura individualista y de cierta desconfianza que caracteriza no solo a los trabajadores del campo sino también a todo el Ecuador. Esto último viene motivado por experiencias pasadas sufridas por los agricultores, haciendo que tengan posiciones negativas hacia la asociación y hacia los liderazgos en ella.

La esperanza del liderazgo para las asociaciones podría estar en las nuevas generaciones, en las que se deberá invertir en el plano educativo para generar líderes, pues el liderazgo se basa en gran parte en el conocimiento. Sin embargo, para entender por qué no han funcionado las capacitaciones en este sector hay que recurrir tanto a la escasa educación de los agricultores (el $60 \%$ de ellos posee un nivel de primaria y tan solo el $10 \%$ ha alcanzado una educación de tercer nivel, tal como indica el Gráfico n`3), como a la falta de articulación de aquéllos con el resto de actores del sector (instituciones gubernamentales, entidades privadas, intermediarios y ONG's).

La no existencia de una adecuada gestión en las asociaciones por ausencia de líderes trae como

\section{${ }_{95}$ TsaFiquim}


consecuencia una falta de tecnificación para mejorar la productividad. Un caso ilustrativo se observó con los minifundios de entre dos y cinco hectáreas de extensión, en los que debido a su reducido tamaño los agricultores toman la decisión de sembrar más productos además del cacao ante la demora de éste en iniciar su periodo productivo (el cacao recién empieza a dar frutos a los tres años, logrando su máxima capacidad a los cinco años), de forma que el campesino diversifica su producción para no depender de un solo producto. Esto, unido al manejo inadecuado del cultivo, hace que la productividad por hectárea se sitúe entre los 15 y los 20 quintales (qq); si bien un agricultor capacitado en el manejo del cacao de la provincia ha conseguido una productividad en este tipo de minifundios de hasta 70 qq/Ha (Ramírez A., 2013).

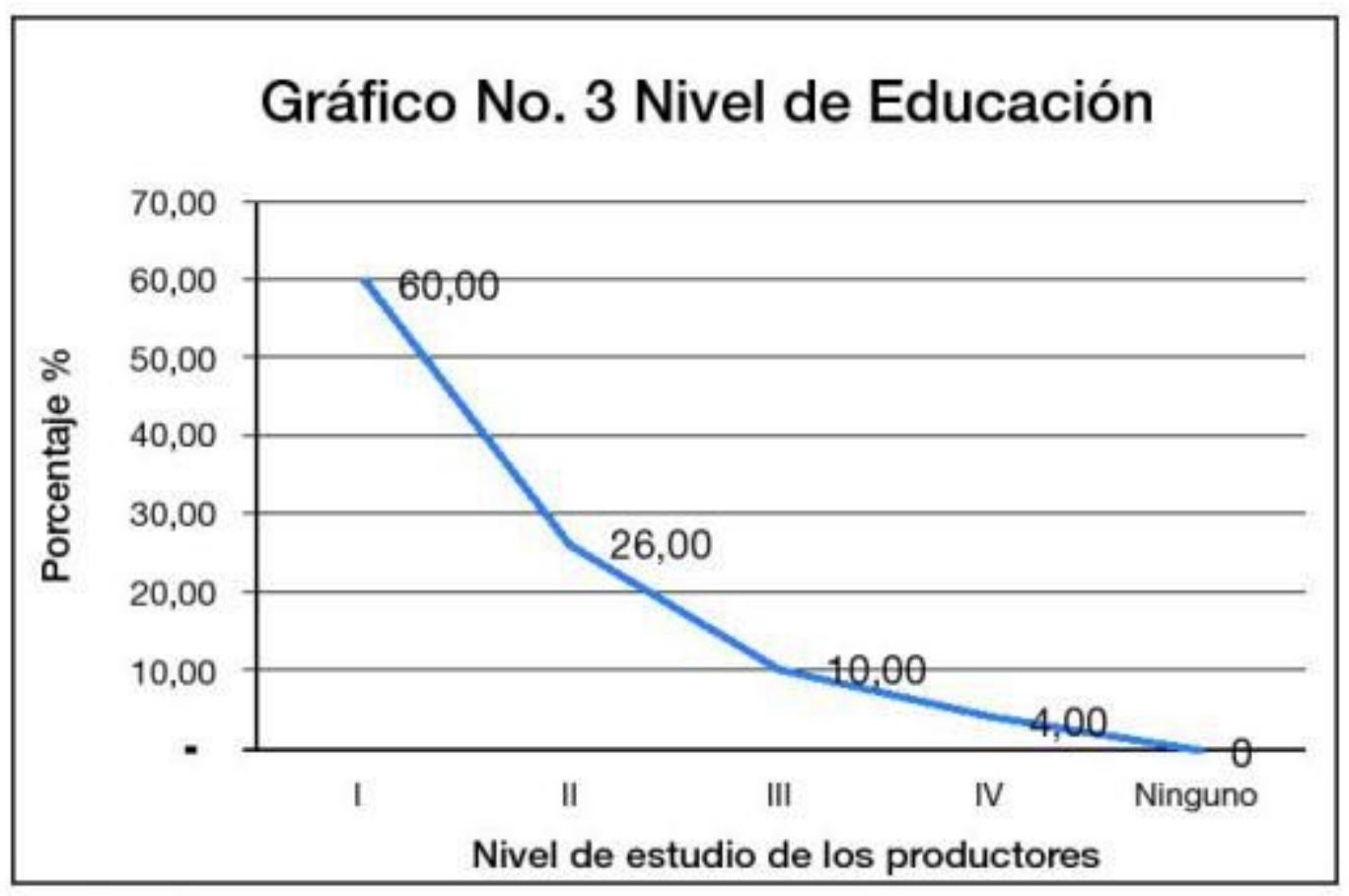

Fuente: Línea Base GAD

Otro efecto de la falta de de liderazgo en las asociaciones objeto de estudio es que sus asociados no han logrado dar un valor agregado al cacao que producen, como pudiera ser su procesado para obtener pasta de cacao, que no requiere de mayor inversión económica. De nuevo aquí el factor educativo de los agricultores es determinante.

\section{Control}

Un primer aspecto a considerar en el control por parte de las asociaciones reside en la ausencia de formación de sus socios en el aspecto administrativo, y las capacitaciones carecen de seguimiento adecuado por parte de las instituciones. Las capacitaciones que el GAD provincial ha efectuado con los agricultores fueron de naturaleza técnica, comercial, pero ninguna fue impartida sobre administración. Este aspecto es importante debido a que el agricultor está sujeto al nuevo sistema de Economía Popular y Solidaria, bajo el cual deben estar en capacidad de saber administrar, ya que se considera una adecuada gestión de las asociaciones como el primer paso para conseguir una mejor calidad de la pepa para luego darle un valor agregado.

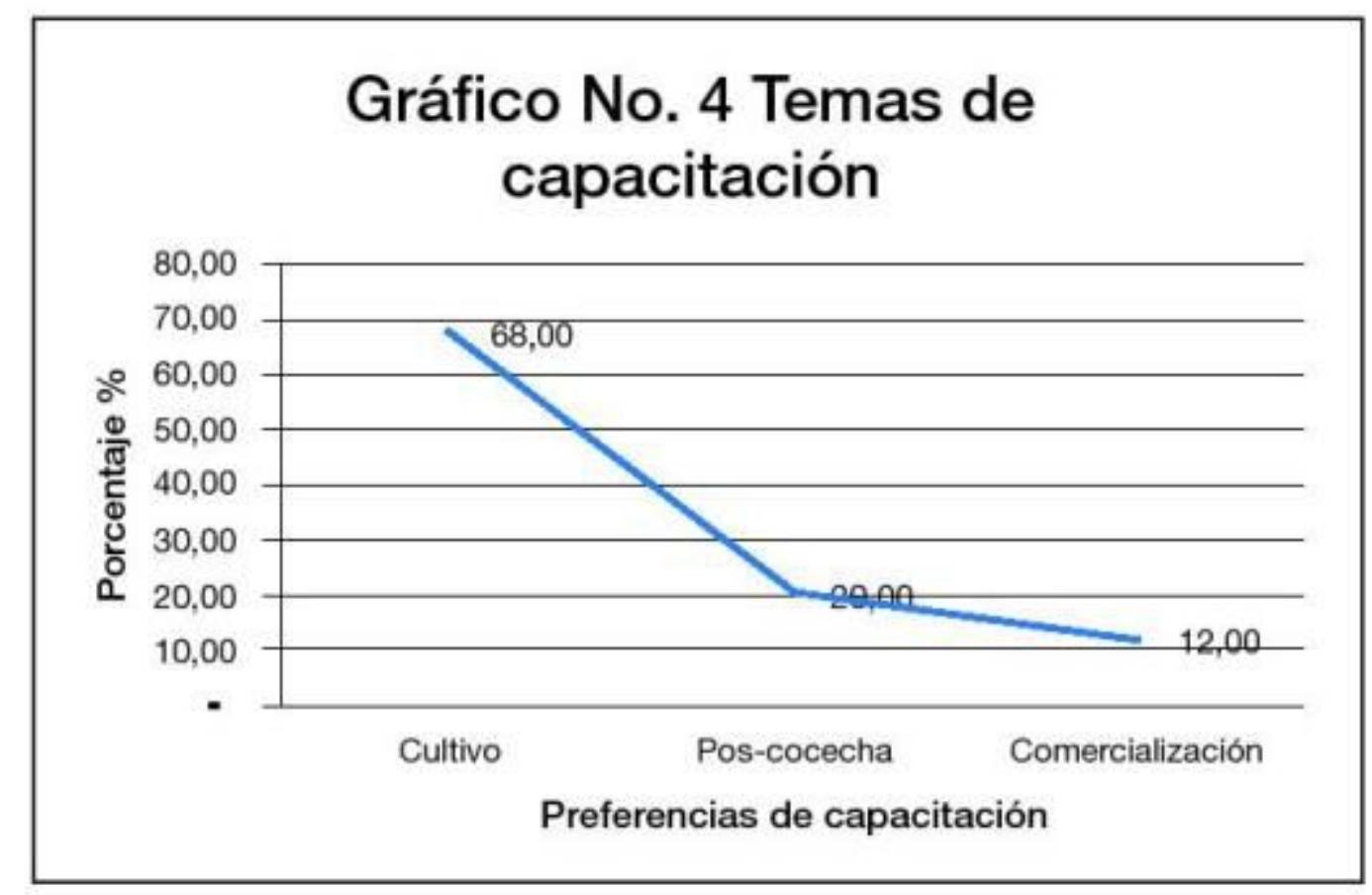

Fuente: Linea Base GAD

La productividad depende también del nivel tecnológico utilizado tanto en el manejo de cosecha como en la poscosecha; en el caso de las asociaciones de Santo Domingo ambos procesos se efectúan de forma rudimentaria. La aplicación de tecnologías también se observa en otros factores como el manejo de clones de plantas adecuados a cada microclima, el aspecto fitosanitario o las podas o la observancia de buenas prácticas en la poscosecha. En la realidad de las asociaciones se ha observado que pese a los intentos del GAD provincial por mejorar la tecnología en esos aspectos, los agricultores se rehúsan cambiar a las variedades de clones adecuados o a utilizar determinados productos fitosanitarios por el coste económico que les supone y la tardanza en obtener réditos de esas acciones.

El control de la eficiencia en la producción de cacao es posible medirla mediante distintos indicadores. Uno puede ser el número de quintales que es entregado al centro de acopio; otro puede ser el volumen de producción por hectárea, que según explotaciones varía entre los $15 \mathrm{qq} / \mathrm{H}^{a}$ hasta $30 \mathrm{qq} / \mathrm{H}^{\mathrm{a}}$; y un tercero bien pudiera ser la calidad de la pepa enviada al centro de acopio. En este último caso la medición de la calidad se 
realiza a través del nivel de humedad de los frutos: por ejemplo, una humedad del $33 \%$ siempre es penalizada con un menor precio para el productor. En la calidad también hay que tomar en consideración la acción de las plagas sobre las pepas, que afectan a su calidad final.

Para ser eficaces en el sector se planifica realizar una marca de la provincia Tsáchila que se oriente al comercio justo, esto se lograra con la ayuda del GAD y la UE.

\section{DISCUSIÓN}

El apoyo del Gobierno nacional a la asociatividad es evidente. Las políticas, lineamientos y estrategias con las instituciones privadas que se están implementado, así como mecanismos adecuados como el comercio justo, sistema basado en la equidad tanto para el productor como para el consumidor, pretenden mejorar el nivel de vida de los productores y de los actores de la Economía Popular y Solidaria, poniendo también atención en el cuidado del medio ambiente.

Una de las cooperaciones a destacar es la que ha puesto en marcha el Gobierno de Alemania a través del programa de Cooperación Técnica GTZ, que apoya al sector agrícola de Santo Domingo en la diversificación de la oferta exportable de 10-15 productos no tradicionales (maracuyá, piña, entre otros) sobre la base de la asociatividad para el lucro solidario.

Las estrategias y políticas están diseñadas para disminuir la pobreza en los pequeños productores agrícolas. En este marco las iniciativas privadas tienen un enfoque ganar-ganar, como ocurre en el Programa Nacional de Negocios Rurales Inclusivos (PRONERI) del MAGAP en conjunto con el Ministerio Coordinador de la Producción, Empleo y Competitividad; o el proyecto de Competitividad Agraria y Desarrollo Rural Sostenible CADERS del MAGAP, cuyo objetivo es articular la política agraria para el fortalecimiento de la asociatividad.

El Ministerio de Agricultura igualmente gestiona el Instituto Nacional de Capacitación Campesina (INCCA), cuyo fin es ofrecer capacitaciones en aspectos técnicos y metodológicos como la conformación de las organizaciones y gremios campesinos. Existen otras iniciativas de carácter privado y también público además de las citadas que podrían ser aprovechadas por las asociaciones de cacaoteros de la provincia de Santo Domingo, pero lamentablemente no ha sido así, por no estar formalizadas muchas de esas asociaciones debido a lo que ya se ha indicado, a su escasa gestión y capacitación. Esto ha impedido concretar más relaciones con instituciones privadas y sobre todo públicas generadas desde el Gobierno, limitándose únicamente a la ayuda de técnicos del GAD provincial, el MAGAP y el INIAP para el manejo del cultivo.

En la investigación realizada se ha constatado el bajo nivel educativo de los agricultores, ejerciendo esto una influencia negativa en la relación entre los propios miembros de las asociaciones, impidiéndoles articularse adecuadamente y generar un mejoramiento de su nivel de vida. Los investigadores consideran adecuada la conformación de una mesa de concertación por parte del GAD Provincial y la UE en la que se propone una estructura organizacional con una proyección estratégica a futuro para las asociaciones cacaoteras de Santo Domingo, pero este modelo exige que estén presentes en esa mesa no sólo los agricultores, sino también el resto de actores del sector, tomando roles de participación pero dirigidos bajo un esquema asociativo de Economía Popular y Solidaria.

\section{CONCLUSIONES}

Pese a que se considera que el cacao es uno de los productos agrícolas con mayor valor en el mercado, su cultivo no ha logrado que los agricultores de las asociaciones de cacaoteros de la provincia de Santo Domingo mejoren sustancialmente su nivel de vida. La carencia de una mayor educación entre los socios de estas entidades provoca la ausencia de líderes en ellas y deficiencias en la gestión de las mismas, en parte atribuidas al poco grado de unión existente entre los socios, lo que deriva el aislamiento físico y la desconfianza ante experiencias asociativas negativas anteriores. Ello representa una dificultad a la hora de planificar todo el proceso de cultivo y manejo del fruto, la tecnificación de las distintas etapas de la producción, la obtención de crédito para precisamente mejorar

\section{${ }_{97}$ TsaFiquil=}


los niveles de producción y la calidad de las pepas e incluso aportar valor añadido al producto, o tener una posición negociadora más fuerte frente a intermediarios y centros de acopio. Por otra parte, la participación del GAD provincial como actor del proceso resultó muy importante en esta experiencia, demostrándose su papel fundamental en el desarrollo futuro de una estructura más fuerte y organizada de las asociaciones, así como de su rol de "cohesionador" de todos los actores involucrados en la cadena de valor del cacao bajo los esquemas de la EPS (Gobierno, productores, comercializadores, empresas privadas, ONG's y gobiernos extranjeros), haciéndose patente la posibilidad real de vínculo estrecho y sistemático entre actores y participantes todos del proceso de producción y comercialización del Cacao en Santo Domingo, lo que a largo plazo tendrá un impacto directo en la superación de los actuales estándares de vida de los productores cacaoteros de la provincia.

\section{AGRADECIMIENTOS}

A los estudiantes que participaron en el levantamiento de la información; a cada uno de los 729 agricultores por colaborar; a la gerente del Proyecto de Mejoramiento de la Cadena Productiva de Cacao y los técnicos de manejo de cacao y asociatividad del Gobierno Autónomo Descentralizado de Santo Domingo de los Tsáchilas; a los dueños de los centros de acopio por la atención prestada a los estudiantes; al presidente de la Asociación de Cacaoteros de Santo Domingo; Msg Ramiro Lapeña y al PhD Fernando Uset.

\section{REFERENCIAS BIBLIOGRÁFICAS}

1. El Comercio (2012) La provincia Tsáchila ocupa el quinto lugar de hectáreas sembradas de cacao en el país. Diario El Comercio. Recuperado 6 de octubre 2013, de http://edicionimpresa.elcomercio.com/ es/220327042

2. Gobierno Autónomo Descentralizado de Santo Domingo (2012). Fortalecimiento de la cadena agroproductiva del cacao, en la provincia de Santo Domingo de los Tsáchilas. Proyecto presentado ante la Delegación de la Unión Europea para Colombia y Ecuador.

3. Instituto Nacional de Estadística y Censos (2013). Censo Nacional Económico. Recuperado el 25 de octubre 2013 de www.ecuadorencifras.gob.ec/ censo-nacional-economico.

4. Luckesh, Robert (2000). Patrones de sustentabilidad. Hacia un meta modelo de instrumentos para el desarrollo sostenible. Proyecto Amazonas 21.

5. Liendo, Mónica G.; Martínez, Adriana M. (2001) Asociatividad. Una alternativa para el desarrollo y Crecimiento de las PYMES. Ponencia presentasa en las Sextas Jornadas "Investigaciones en la Facultad" de Ciencias Económicas y Estadística, Universidad Nacional de Rosario. Rosario, Argentina.

6. Narváez M., Fernández G., Gutiérrez C., Revilla J. y Pérez C. (2009) Asociatividad empresarial: un modelo para el fortalecimiento de la Pyme en Paraguaná, mayo-agosto. Redalyc, 9(2). 157-166 Recuperado de la base de datos Academic Search Premier. EBSCO Host

7. Ramírez A., Ángel (2013) Los secretos para el cultivo del cacao. Incremente su productividad. EcuadorSanto Domingo: Ángel Ramírez.

8. Rosales Ramón (1997). La asociatividad como estrategia de fortalecimiento de las Pymes. II Reunión del Foro Regional sobre Política Industrial, realizado del 30 de julio al 1 de agosto de 1997, en la sede del SELA. 